\title{
Pharmarcogenetic Mechanism of ACE I/D Polymorphism Adversely Responding to ACE Inhibitors in Regulating the ACE Promoter Activity in Neurons
}

\author{
Yuan-Han Yang ${ }^{1,2,3}$, Hsueh-Wei Chang ${ }^{4,5}$, Wei-Chiao Chang ${ }^{6}$, Yu-Cheng Shih ${ }^{7}$, Ke-Li Tsai ${ }^{8}$, I Chien ${ }^{9}$ and \\ Shyh-Jong $\mathrm{Wu}^{9}$ \\ 1. Department of Neurology, Kaohsiung Municipal Ta-Tung Hospital, Kaohsiung Medical University, Kaohsiung, Taiwan, R.O.C. \\ 2. Department of Master's Program in Neurology, Faculty of Medicine, Kaohsiung Medical University, Kaohsiung, Taiwan, R.O.C. \\ 3. Department of Neurology, Kaohsiung Medical University Hospital, Kaohsiung Medical University, Kaohsiung, Taiwan, R.O.C. \\ 4. Department of Biomedical Science and Environmental Biology, Kaohsiung Medical University, Kaohsiung, Taiwan, R.O.C. \\ 5. Institute of Medical Science and Technology, National Sun Yat-Sen University, Kaohsiung, Taiwan, R.O.C. \\ 6. Department of Clinical Pharmacy, School of Pharmacy, Taipei Medical University, Taipei City, Taiwan, R.O.C. \\ 7. School of Medicine, College of Medicine, Kaohsiung Medical University, Kaohsiung, Taiwan, R.O.C. \\ 8. Department of Physiology, College of Medicine, Kaohsiung Medical University, Kaohsiung, Taiwan, R.O.C. \\ 9. Department of Medical Laboratory Science and Biotechnology, Kaohsiung Medical University, Kaohsiung, Taiwan, R.O.C.
}

\begin{abstract}
The ACE (angiotensin converting enzyme) inhibitors are not only drugs widely prescribed drugs in cardiovascular diseases, but also potentially therapeutic agents in dementia. Based on the findings that the ACE inhibitors could activate the c-Jun N-terminal kinase signal to increase the ACE gene expression and that the Alu element of the human ACE gene involved in regulating ACE promoter activity, we aimed to investigate whether there are different pharmacogenetic responses of ACE I/D polymorphism to the ACE inhibitors in neurons. The three reporter vectors, pACEpro(f)-SEAP, p-I-ACEpro-SEAP, and p-D-ACEpro-SEAP were used to examine the transcriptional activity of the vectors responding to the lisinopril treatment using a transient-transfection method in SH-SY5Y cells. Our results showed that lisinopril increased the promoter activity of an ACE gene by $16.7 \%$. Additionally, we found the lisinopril enhanced the ACE promoter activity of the I-form vector by $17.2 \%$, but adversely reduced that of the D-form vector by $16.8 \%$, as compared with the respective control without the lisinopril treatment. Firstly, our findings had proved that the I/D polymorphism of ACE gene contrarily responds to the ACE inhibitors in regulating the ACE expression in neurons, which provide a novel insight suggesting genetic testing to tailor the treatment regimens in AD (Alzheimer's disease) patients.
\end{abstract}

Key words: ACE inhibitors, ACE I/D polymorphism, Alzheimer's disease, pharmacogenetics, promoter activity.

\section{Introduction}

The ACE (angiotensin converting enzyme) inhibitors are used extensively for the treatment of CVD (cardiovascular diseases) and kidney diseases to lower the risk of cardiovascular events and kidney failure [1]. Beyond their primary therapeutic goals,

Corresponding author: Shyh-Jong Wu, Ph.D., research fields: the genetics of dementia, diabetes and cardiovascular diseases.
ACE inhibitors are also considered as potentially therapeutic agents in dementia as per several clinical studies [2-5], especially for the centrally active ACE inhibitors [6]. Two large clinical trials indicated that the ACE inhibitors could secondarily reduce an incidence of cognitive impairment and the rate of cognitive decline, i.e., the PROGRESS (Perindopril Protection against Recurrent Stroke Study) [7] and the SYST-EUR (Systolic Hypertension in Europe) 
trial [8]. The similar pharmacologic effects of the ACE inhibitors on cognitive function were also found in recent clinical trials [9-11] and in animal models $[12,13]$. It is noteworthy that some studies denoted that the direct effects of the ACE inhibitors to lower the risk of developing dementia could be independent from the controlling of cardiovascular disease and blood pressure control [2, 4, 14]. Nevertheless, these inconsistent results have also been reported in other studies [4, 15-18]. Whatever the positive or negative results, no clear mechanisms have been delineated.

Being the main target of the ACE inhibitors, ACE is a well-known zinc-containing metallopeptidase that plays a key role in the RAS (renin-angiotensin system) and performs diverse physiological functions in many tissues [19]. In fact, the local ACE expressed in brain tissue has also been identified in different neuron cells [20], and the increased ACE protein level and activity in the brain of $\mathrm{AD}$ (Alzheimer's disease) patients have been found in many studies [21-23]. In a recent multifaceted review [24], the principal enzymatic functions of ACE, i.e., converting decapeptide ANG I (Angiotensin I) into octapeptide ANG II (Angiotensin II) and hydrolyzing A $\beta 1-42$ peptide to small amyloid peptide [25, 26], have been suggested to underpin the association with the risk of AD. However, the exact mechanism of how ACE is involved in the pathogenesis of $\mathrm{AD}$ is still a challenging fact to elucidate. Interestingly, and peculiarly noteworthy, the membrane-bound ACE has been found to function as a receptor that can be activated by the ACE inhibitors [27] or sheer stress [28] to induce the ACE signaling cascade, which in turn regulates the expression of the ACE protein itself. Therefore, it needs more experimental strategies to explore the unidentified role of ACE in the pathogenesis of $\mathrm{AD}$, and the possible functions of the ACE inhibitors.

To explore the plausible pathophysiological role of $\mathrm{ACE}$ in contributing to $\mathrm{AD}$, the genetic marker of the
ACE I/D, an I (insertion) or D (deletion) of the Alu element located in Intron 16 has also been extensively examined in association with the risk for AD. The I allele of the ACE gene has been indicated as a potential susceptibility marker for $\mathrm{AD}$ in many previous studies [29-32]. However, some contradicting results keep the previous conclusions as pending [33]. In pharmacogenetic testing, a side study of the PROGRESS trail aimed to observe whether the ACE $\mathrm{I} / \mathrm{D}$ influences the treatment response of macrovascular events and dementia to the ACE inhibitors for patients with a history of cerebrovascular disease, but this study provides no evidence that knowledge of ACE genotype is useful for predicting either the risk of disease or the benefits of perindopril-based blood pressure-lowering treatment [34]. This is a limited trail to test the cognitive function response to the ACE inhibitors according to the ACE I/D polymorphism of individuals. However, it is noteworthy that this study mainly focused on individuals with a history of a stroke, or a transient ischemic attack rather than specifying on $\mathrm{AD}$ or any characteristic types of dementia. Another similar pharmacogenetic cohort study indicated that the 6AG and M235T polymorphisms in the AGT gene were associated with a cognitive function response to the ACE inhibitors in a group of elderly and healthy participants (the Health, Aging and Body Composition Study) [35], but it was not applied for the ACE I/D genotype. Whereas AD is a complex polygenic disease, many candidate genes may contribute to each partial effect on the pathogenesis to the development of AD. Thus, it might be too early to reach a conclusion that the ACE I/D polymorphism is not associated with a cognitive improvement response to the ACE inhibitors by limited epidemiological studies.

Based on our recent report that the Alu element in the intron 16 of the ACE gene is involved in regulating the activity of the ACE promoter in neurons [36], the present study further aimed to examine whether the 
ACE I/D polymorphism respectively responds to the ACE inhibitors in regulating the ACE prompter activity using reporter activity assay in SH-SY5Y cells. To unravel the feasible mechanisms of the pharmacogenetic response of ACE I/D to the ACE inhibitors in neurons will provide a helpful insight for further clinical trials.

\section{Materials and Methods}

\subsection{Construction of the Luciferase Reporter Vectors}

The detail of the three reporter vectors used in the present study was described in our previous work [36] and the modified schematic diagrams are illustrated in Fig. 1. Briefly, the backbone of our reporter vectors is the pSEAP-Basic2 vector (pSEAP-Bas) (BD Biosciences Clontech, Palo Alto, CA, USA). The promoter fragment of the human ACE $(-760 \sim+130)$ including many proved binding elements was amplified by a PCR (polymerase chain reaction) and first cloned into the multiple cloning site of the pSEAP-Bas vector using EcoRI restriction enzyme. The strain with forward direction of the ACE promoter,
pACEpro(f)-SEAP vector, were obtained. The I allele and the D allele fragments of human ACE Intron 16 were identified and amplified by the PCR, then $479 \mathrm{bp}$ of I form fragment (with Alu element) and 192 bp of D form fragment were extracted and inserted between the Hind III and Xho I sites in front of the ACE promoter region in the pACEpro(f)-SEAP vector to obtain the p-I-ACEpro-SEAP vector and the p-D-ACEpro-SEAP vector, respectively. All these reporter constructs were verified by completely sequencing. The primers used for the PCR and DNA sequencing of the I and D fragments were as follows: forward primer, 5'-ACCGCTCGAGCTGGAGAGCCACTCCCATCC TTTCT-3'; reverse primer, 5'-CTAAAAGCTTGACG TGGCCATCACATTCGTCAGAT-3'.

\subsection{Cell Viability Assay}

Lisinopril, a highly efficient inhibitor of ACE, and OA (okadaic acid) were purchased from Sigma-Aldrich, Inc (Saint Louis, USA). OA, a protein PP2A (Phosphatase-2A) inhibitor, could induce hyperphosphorylation of tau protein and cause neuronal

\section{pSEAP-Bas}

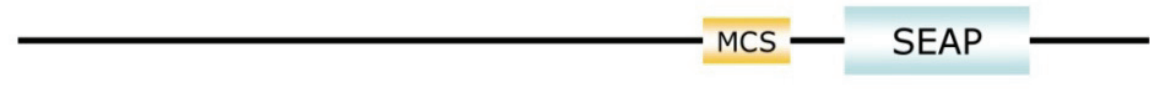

pACEpro(f)-SEAP

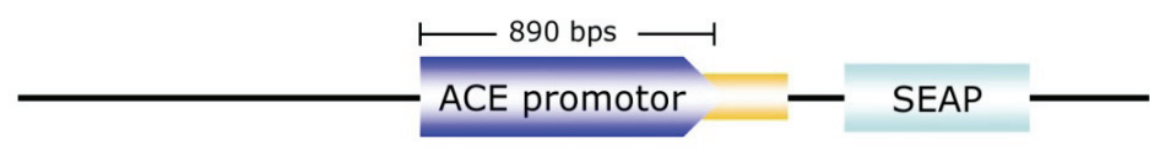

p-I-ACEpro-SEAP

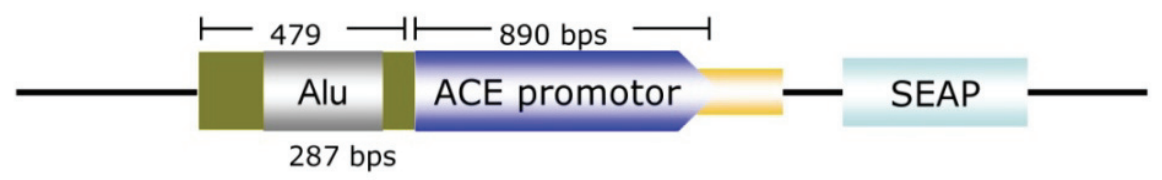

p-D-ACEpro-SEAP

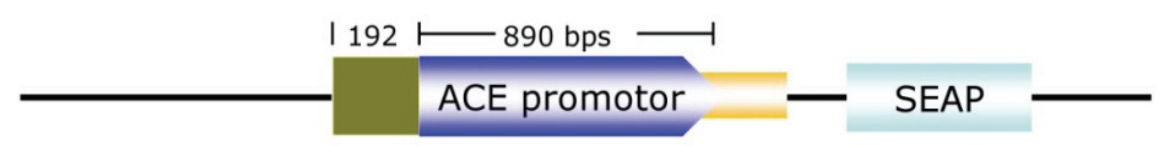

Fig. 1 Schematic diagrams of the reporter vectors in the present study modified from the figure in our previous work [36]. 
cell death in culture, thus it was selected as a positive control for the cell viability assay. Cell viability was detected using a PrestoBlue $\mathrm{TM}^{\mathrm{TM}}$ kit (Invitrogen, Carlsbad, USA). Prestoblue is quickly reduced by metabolically active cells, providing a quantitative measurement of viability and cytotoxicity. SH-SY5Y cells $\left(1 \times 10^{4}\right)$ were seeded in 96-well plates overnight before the experiment, and the cells were then treated with a serial working concentration of lisinopril (i.e., $10 \mu \mathrm{M}, 100 \mu \mathrm{M}, 1 \mathrm{mM}$ and $10 \mathrm{mM}$ ) or $30 \mathrm{nM} \mathrm{OA}$. After $24 \mathrm{~h}$ incubation, the cells were rinsed with PBS solution, and $10 \mu \mathrm{L}$ of prestoblue reagent was loaded. After 30 min of incubation at $37^{\circ} \mathrm{C}$, the cell viability was assessed by measuring the fluorescence of the PrestoBlue ${ }^{\mathrm{TM}}$ reaction (excitation at $515 \mathrm{~nm}$, emission at $590 \mathrm{~nm}$ ).

\subsection{Cell Culture and Transient Transfection}

The maintenance of SH-SY5Y cells, a neuroblastoma cell line, and transient transfection examination were also described in our previous work [36]. Briefly, the cells were maintained in MEM/F-12 (1:1) containing 10\% fetal bovine serum in a humidified atmosphere of $95 \%$ air and $5 \% \mathrm{CO}_{2}$ at $37^{\circ} \mathrm{C}$. All experiments of the cell line were carried out within passage 12 to maintain uniform condition. The plasmid DNAs for transfection were prepared using QIAGEN columns (QIAGEN GmbH, Hilden, Germany). The cells were freshly subcultured at a density of $2 \times 10^{5}$ cells on 6 -well cassettes and were transfected using a transient liposome co-transfection protocol (TransFast ${ }^{\mathrm{TM}}$ Transfection Reagent, Promega, Charbonniere-les-bains, France) with $2.4 \mu \mathrm{g}$ of reporter plasmids and $0.6 \mu \mathrm{g}$ of pGL4 Luciferase Reporter Vectors for firefly luciferase gene expression as an internal control to normalize efficiency of transfection. All samples were run in quadruplicate in at least four different experiments.

\subsection{Reporter Assay}

The day after transfection, the medium was changed to a completely fresh medium with the same volume per well. After another $48 \mathrm{~h}$, the conditioned cell culture medium was collected for measurement of SEAP activity of each construct by a Great EscAPe ${ }^{\mathrm{TM}}$ SEAP Chemiluminescence Detection kit (BD Biosciences Clontech) and Luminometer (Labsystems Luminoskan). The luciferase assay of pGL4 firefly luciferase in cell lysate was performed according to the manufacturer's protocol form the commercial kit (Promega).

\subsection{Statistics}

Two-way ANOVA (analysis of variance) and Student's $t$-test were performed to compare the expression activity of reporter constructs in at least four independent transfection experiments as per the previous report [37]. Data are expressed as means $\pm \mathrm{SE}$, and values of $p<0.05$ were considered statistically significant.

\section{Results}

\subsection{The Effect of Lisinopril on the Cell Viability of SH-SY5Y Cells}

Lisinopril, a centrally active ACE inhibitor with a high specificity to ACE, was selected to examine whether the ACE promoter responded to the ACE inhibitors in neuron. First, we analyzed the dose-dependent effects of lisinopril on the cell viability of SH-SY5Y cells using variant concentrations of lisinopril from $10 \mu \mathrm{M}$ to $10 \mathrm{mM}$ (Fig. 2). Our results showed that even the high concentration of lisinopril $(10 \mathrm{mM})$ did not affect the cell viability of SH-SY5Y cells, as compared to $40 \%$ of cell viability reduction in the treatment of $30 \mathrm{nM} \mathrm{OA}$. In order to bind the membrane-bound ACE completely, $100 \mu \mathrm{M}$ of lisinopril was used to perform the following reporter assays of the ACE promoter activity.

\subsection{Lisinopril Enhances the ACE Promoter Activity}

The promoter activity of the pACEpro(f)-SEAP 
vector was assayed after the transfected cells were exposed to plus or minus $100 \mu \mathrm{M}$ of lisinopril for $24 \mathrm{~h}$. We found that lisinopril significantly up-regulated the promoter activity of the pACEpro(f)-SEAP vector by approximately $17 \%(p<0.01)$ (Fig. 3 and Table 1), which is consistent with the previous finding that the ACE gene was increased by the ACE inhibitors activation [38].

\subsection{Contrary Response of I and D Form Fragment to Lisinopril Treatment}

Furthermore, SH-SY5Y cells were transiently transfected with p-I-ACEpro-SEAP or p-D-ACEpro-SEAP respectively, and then the cells were parallelly treated with or without $100 \mu \mathrm{M}$ of lisinopril for $24 \mathrm{~h}$ in each set of the experiment. The reporter assay showed that the promoter activity of p-I-ACEpro-SEAP was increased to $117 \%$ responding to lisinopril treating in comparison to the control group (Fig. 4 and Table 2). In contrast, we found that lisinopril suppressed approximately $18 \%$ of the ACE promoter activity in the cells transfected with p-D-ACEpro-SEAP (Fig. 5 and Table 3).

Further, we wanted to compare the relative reporter activity of I form and D form vectors responding to lisinopril. There, we designated the promoter activity of the pACEpro(f)-SEAP group without treating lisinopril as $100 \%$, and then merged the present results above and our previous results (Fig. 6a) [36] into one figure (Fig. 6b). After recalculating the relative percentage of the promoter activity of the three reporter vectors in SH-Sy5Y cells incubated with or without lisinopril, we found that the I form reporter vector (p-I-ACEpro-SEAP) showed 2-fold higher promoter activity in responding to lisinopril as compared with the D form vector (p-D-ACEpro-SEAP) in SH-SY5Y cells.

\section{Discussion}

In the present study, we first demonstrate the mechanisms that the ACE inhibitors could increase the promoter activity of the ACE gene in neurons, and that I and D form alleles exert an adverse response to lisinopril in regulating the ACE promoter activity. We propose that the binding of lisinopril to membrane-bound ACE might trigger phosphorylation of c-Jun and the activation of AP-1 (intracellular signaling cascade) that leads to an increase of the ACE

\section{SH-Sy5y}

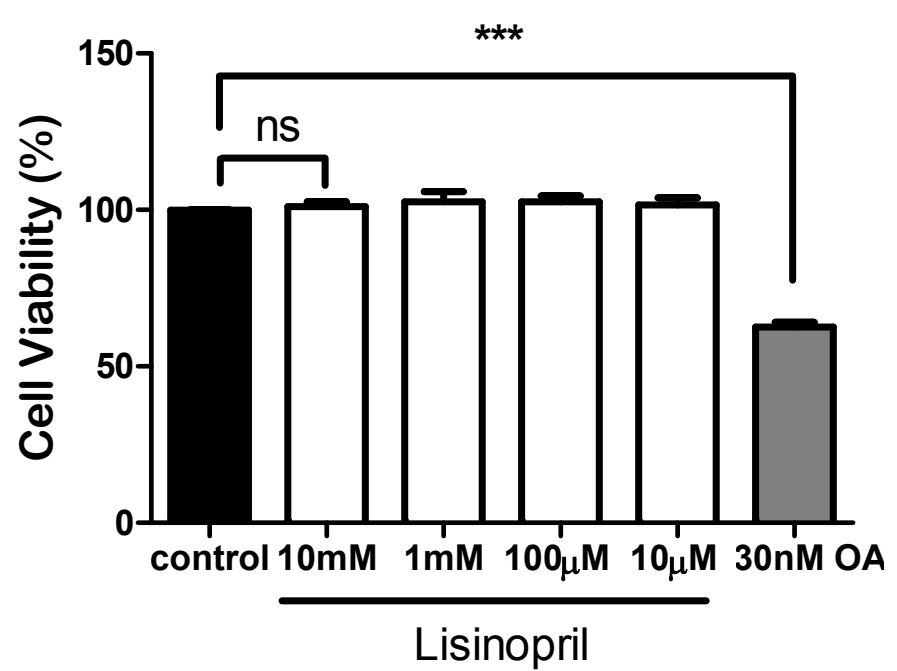

Fig. 2 Effects of lisinopril on the cell viability of SH-SY5Y cells. SH-SY5Y cells were stimulated with lisinopril (10 $\mu$ M, $100 \mu \mathrm{M}, 1 \mathrm{mM}$ or $10 \mathrm{mM}$ ) or $30 \mathrm{nM} \mathrm{OA}$, respectively. After $24 \mathrm{~h}$, the cell viability was detected by prestoblue reagent. The control group without lisinopril or OA treatment is designated as $100 \%$. Data represent the mean \pm SEM of six independent experiments.

"ns": no significant; “***”: $p<0.001$ relative to control group. 


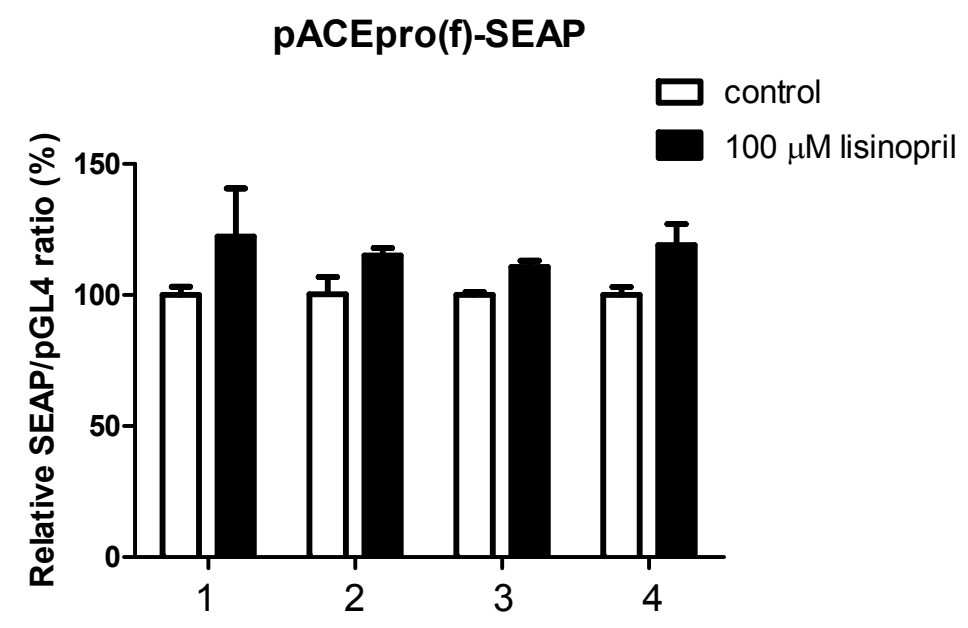

(a)

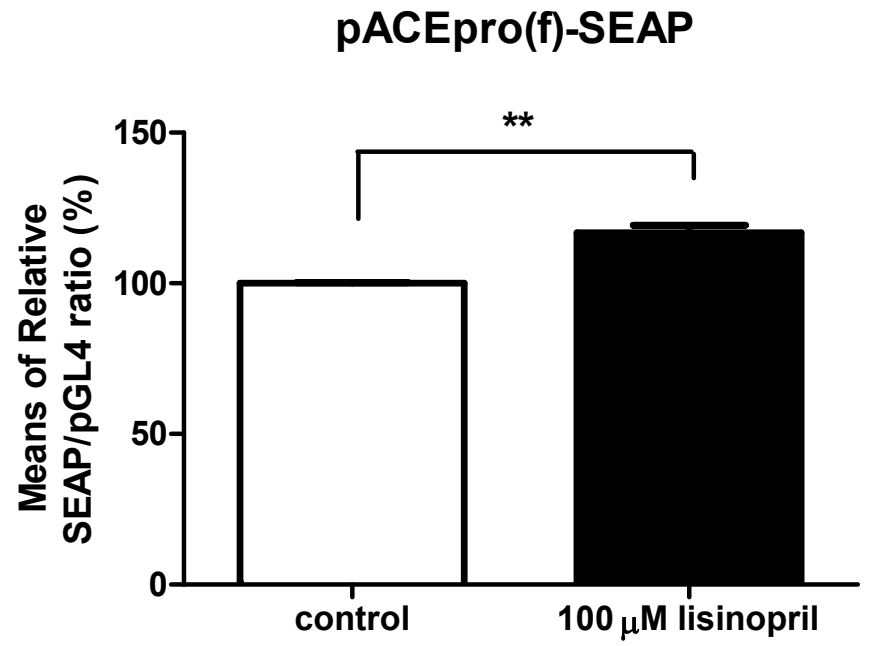

(b)

Fig. 3 Effects of lisinopril on regulating the transcriptional activity of human ACE promoter in SH-SY5Y cells: (a) each relative SEAP/pGL-4 ratio of four independent experiments when the cells were transfected with pACEpro(f)-SEAP vector and treated with or without $100 \mu \mathrm{M}$ lisinopril. For each construct, the experiments were performed in quadruplicate; (b) mean relative ratios of all four independent experiments from Fig. 3a. The control group without treating lisinopril is designated as $100 \%$, and the values are expressed with standard error.

“**”: $p<0.01$ relative to control group.

Table 1 The statistic results from two-way ANOVA analysis in Fig. 3.

\begin{tabular}{lllll}
\hline Effect & & Mean & & Difference \\
\hline Control & & 100.1 & - & - \\
$100 \mu \mathrm{M}$ lisinopril & & 116.8 & - & +16.67 \\
Source of variation & $\mathrm{DF}$ & $\mathrm{MS}$ & $\mathrm{F}$ & $\mathrm{P}$ \\
Residual & 16 & 179.8 & - & - \\
Experiment & 3 & 37.61 & 0.2092 & 0.8885 \\
Control vs. $100 \mu \mathrm{M}$ lisinopril & 1 & 1667 & 9.272 & 0.0077 \\
Interaction & 3 & 38.78 & 0.2157 & 0.884 \\
\hline
\end{tabular}




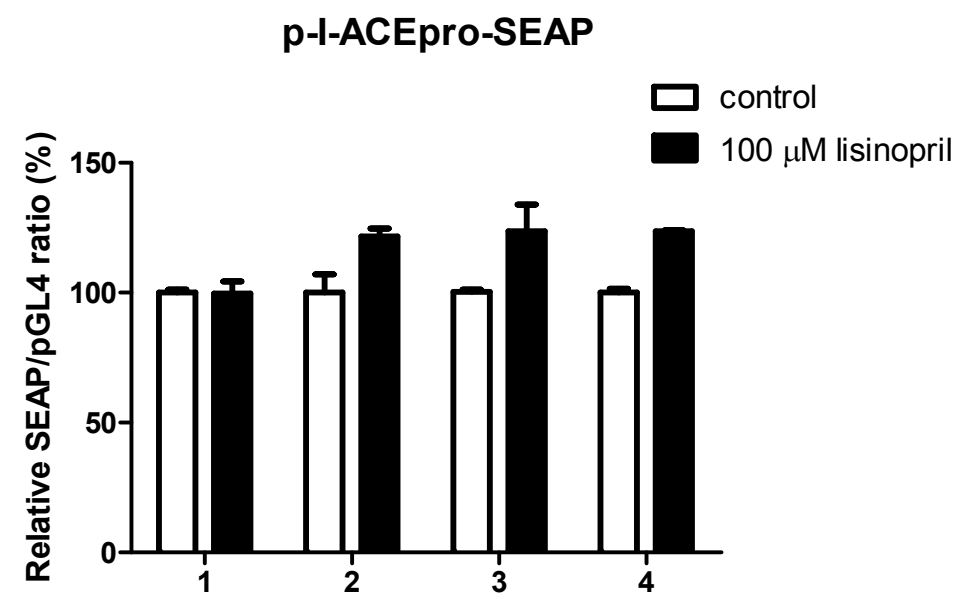

(a)

\section{p-I-ACEpro-SEAP}

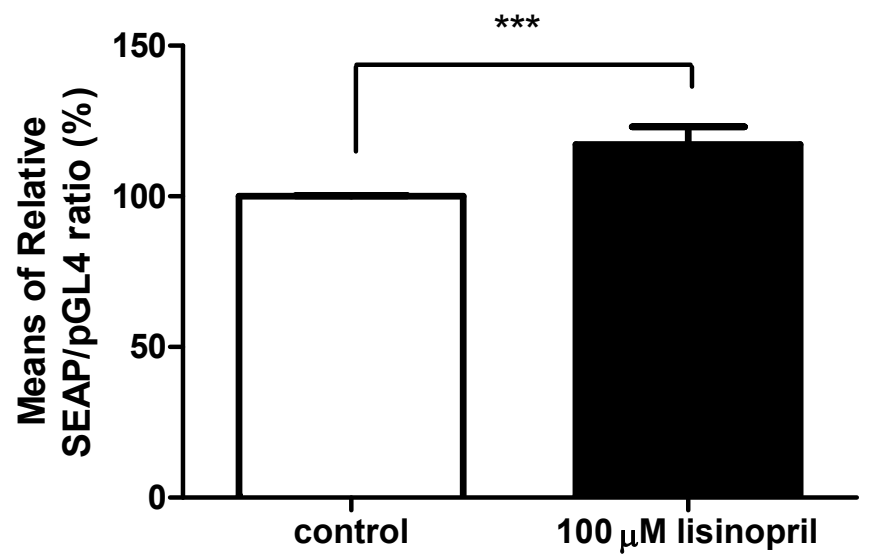

(b)

Fig. 4 Effects of lisinopril on regulating the transcriptional activity of p-I-ACEpro-SEAP in SH-SY5Y cells: (a) each relative SEAP/pGL-4 ratio of four independent experiments when the cells were transfected with pACEpro(f)-SEAP vector and treated with or without $100 \mu \mathrm{M}$ lisinopril. For each construct, the experiments were performed in quadruplicate; (b) mean relative ratio of all four independent experiments from Fig. 4a. The control group without treating lisinopril is designated as $100 \%$, and the values are expressed with standard error.

“***”: $p<0.001$ relative to control group.

Table 2 The statistic results from two-way ANOVA analysis in Fig. 4.

\begin{tabular}{lllll}
\hline Effect & & Mean & Difference \\
\hline Control & & 100.1 & - & - \\
$100 \mu \mathrm{M}$ lisinopril & & 117.2 & - & +17.2 \\
Source of variation & $\mathrm{DF}$ & $\mathrm{MS}$ & $\mathrm{F}$ & $\mathrm{P}$ \\
Residual & 16 & 71.21 & - & - \\
Experiment & 3 & 207.7 & 2.917 & 0.0662 \\
Control vs. $100 \mu \mathrm{M}$ lisinopril & 1 & 1751 & 24.59 & 0.0001 \\
Interaction & 3 & 203.4 & 2.856 & 0.0699 \\
\hline
\end{tabular}




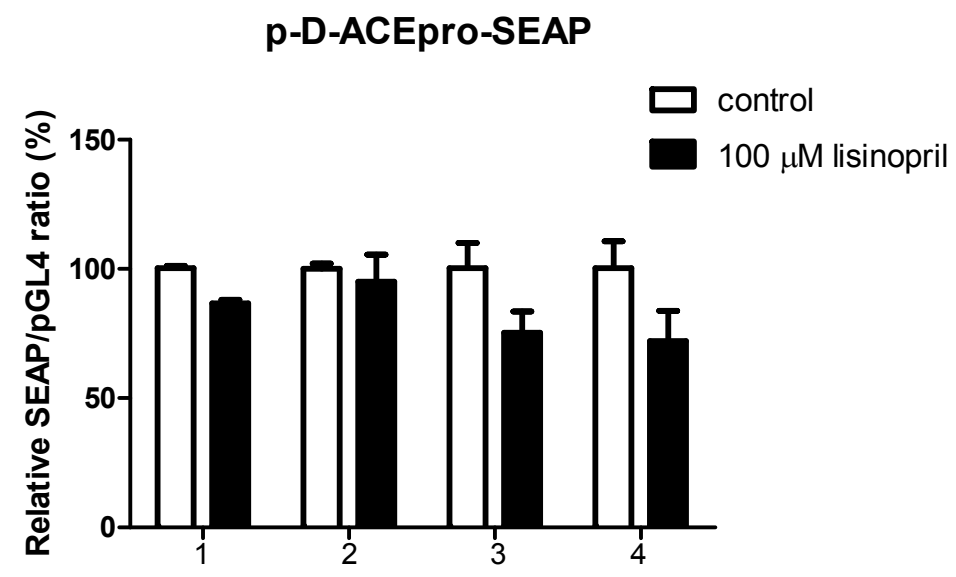

(a)

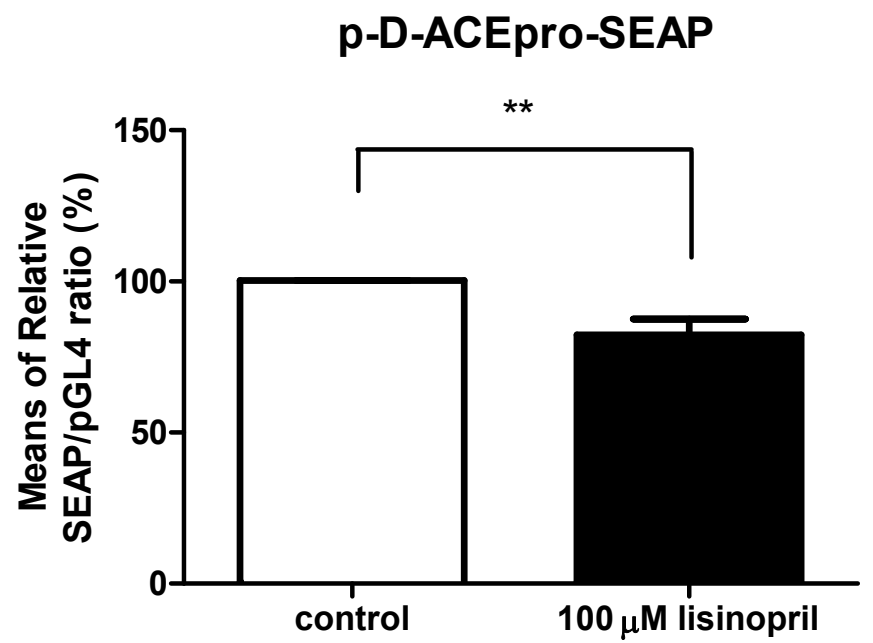

(b)

Fig. 5 Effects of lisinopril on regulating the transcriptional activity of p-D-ACEpro-SEAP in SH-SY5Y cells: (a) each relative SEAP/pGL-4 ratio of four independent experiments when the cells were transfected with pACEpro(f)-SEAP vector and treated with or without $100 \mu \mathrm{M}$ lisinopril. For each construct, the experiments were performed in quadruplicate; (b) mean relative ratio of all four independent experiments from Fig. 5a. The control group without treating lisinopril is designated as $100 \%$, and the values are expressed with standard error.

“**”: $p<0.01$ relative to control group.

Table 3 The statistic results from two-way ANOVA analysis in Fig. 5.

\begin{tabular}{lllll}
\hline Effect & & Mean & & Difference \\
\hline Control & & 100.3 & - & - \\
$100 \mu \mathrm{M}$ lisinopril & & 82.25 & - & -18.00 \\
Source of variation & $\mathrm{DF}$ & $\mathrm{MS}$ & $\mathrm{F}$ & $\mathrm{P}$ \\
Residual & 16 & 197.2 & - & - \\
Experiment & 3 & 163.3 & 0.8279 & 0.4977 \\
Control vs. $100 \mu \mathrm{M}$ lisinopril & 1 & 1944 & 9.858 & 0.0063 \\
Interaction & 3 & 171.8 & 0.871 & 0.4765 \\
\hline
\end{tabular}




\section{Relative ACE promoter activity}

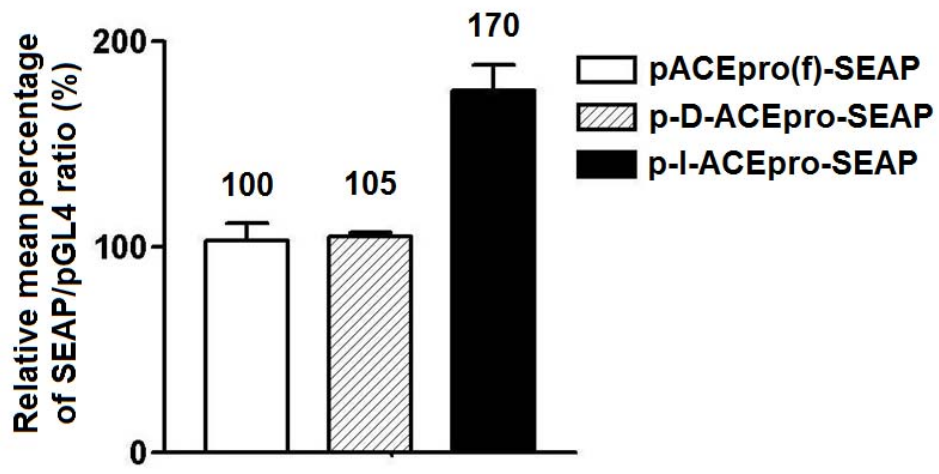

(a)

Relative ACE promoter activity

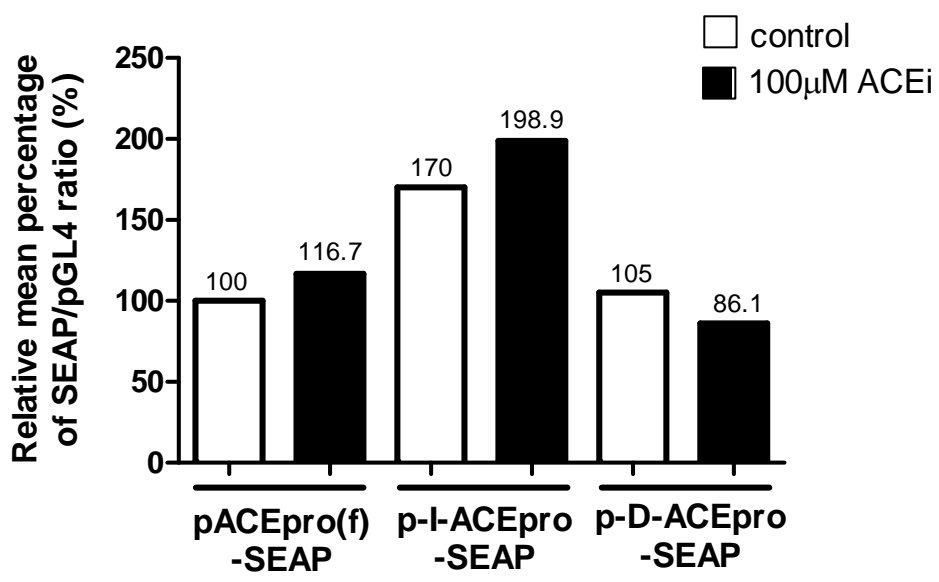

(b)

Fig. 6 The relative response of $I$ and $D$ form to ACE inhibitor in ACE promoter activation: (a) to represent the relative percentage of three relative ACE promoter activities when the transfected cells were not treated with lisinopril, which was modified from Fig. 2 of our previous report [36]; (b) to compare the relative promoter activities of the three vectors in responding to lisinopril, we recalculated the relative percentage of their promoter activities from the results in Figs. 3-5 and 6a. The basal promoter activity of pACEpro(f)-SEAP without lisinoril treatment is designed as $100 \%$ control.

expression as previously reported [39]. Pharmacogenetic testing brings the potential for personalized therapeutic strategies not only to improve health outcomes and efficacy of prescribed medications but also reduce the cost of care. However, there is substantial debate about the clinical utility of pharmacogenetic testing, due in part to the lack of direct evidence or defined mechanisms. Presently, no clinical pharmacogenetic practice is currently applied for $\mathrm{AD}$ treatment and even for cardiovascular medicines [40]. Herein, our study first provides the direct molecular evidence that I/D polymorphism in
Intron 16 of an ACE gene may play a vital role in adversely responding to the ACE inhibitors in regulating the ACE expression in neurons.

It is noteworthy that the pharmacological role and plausible mechanism of ACE inhibitors in AD remain unsolved paradoxes, although Kehoe and Wilcock thoughtfully discussed this issue in their review article based upon the clinical trials and laboratory-based findings [41]. They indicated that the introduction of an ACE inhibitor in order to block the ACE activity might not only reduce the benefits to $\mathrm{A} \beta$ degradation $[42,43]$, but also decrease the potential 
harm of Angiotensin II action on either acetylcholine release or hypertension. In addition, the inhibition of an ACE activity in the brain has also been reported to associate with cognitive improvement via increasing substance $\mathrm{P}$, diminishing inflammatory cytokines, suppressing reactive oxygen species generation, or improving cerebral blood flow $[2,4,6,12]$. In addition to the well-known functions of an ACE enzyme as mentioned above, the physiological implications of ACE continue to expand, in which ACE has been found to exert many other functions than is usual and is localized in different brain tissues. For instance, Paul et al. [44] indicated that "intracellular" ACE converting ANG I to intracellular ANG II in brain neurons may facilitate learning and memory. A functional RAS in mitochondria was demonstrated to have a role in nitric oxide production in many tissues including neurons [45]. Moreover, Bernstein et al. recently presented that overexpression of ACE in myelomonocytes could prevent Alzheimer's-like cognitive decline through degrading $\mathrm{A} \beta$ and enhancing immunity functions [46]. Taken together, it keeps pending to conclude if the induction of an ACE expression by the ACE inhibitors in neurons is beneficial to cognitive decline or otherwise. Thus, to further clarify the pharmacological role of the ACE inhibitors in increasing its target, an ACE protein could be challengeable but very helpful to understand the clinical application of the ACE inhibitors.

According to our present findings, lisinopril positively enhances I allele-increased promoter activity of the ACE gene because of the Alu element. In contrast, $\mathrm{D}$ allele seems to restrict the promoter activity responding to lisinopril in neurons. Due to the paradoxical hypothesis of the ACE function relevant with $\mathrm{AD}$ pathogenesis as discussed above, there would be two novel contrary perspectives to interpret our results: (1) if the increased ANG II is the main pathogenesis consideration for some subgroups of $\mathrm{AD}$ patients, then the individuals with $\mathrm{D}$ allele genotype in the subgroups would be suggested to have the introduction of the ACE inhibitors to restrain their $\mathrm{ACE}$ expression; (2) in contrast, if the clearance of $A \beta$ aggregation is the priority for some subpopulations of AD patients, then it might be beneficial for the individuals with I allele to take the ACE inhibitors in order to produce more ACE molecules. Therefore, before an associated pharmacogenetic trail of an ACE I/D with better cognitive response to the ACE inhibitors in $\mathrm{AD}$, we suggest in advance an effective strategy to subgroup the pathological type of $\mathrm{AD}$ individuals.

However, the inconsistently associated results of the ACE I/D polymorphism with a specific disease (e.g., CVD or AD) have led to some arguable issues existing for more than a decade. First, the main question is that none of the available direct evidence has been provided to delineate the definite molecular mechanism by which $\mathrm{D}$ allele was pointed out to be associated with a higher ACE activity or concentration. The second vital question is whether the secreted ACE in serum could represent the amount of all ACE protein expressed from all tissues of the individual. Recently, a study provided an important evidence that the secreted ACE in human serum is very minor part of expressed ACE in comparison to membrane-bound ACE [47]. Besides, Fagyas and his colleagues [48, 49] showed that most of the secreted ACE in body fluid are blocked by natural ACE inhibitors in serum (e.g., serum albumins). Moreover, according to Chattopadhyay's report [50], the secreted form of ACE can not replace the tissue-bound ACE for maintaining normal blood pressure. According to the above, it might not be the best investigative strategy to use the concentration of serum ACE to represent the function of systemic ACE, and it might be even unwarrantable to use serum ACE to interpret the pathophysiological role of a specific local ACE expression. Therefore, those previous studies that examined the association between the serum ACE concentration and chronic diseases might need to be 
reevaluated. Conceptually, it would be more questionable to use serum ACE concentration, but not CSF ACE, to explore the association of an ACE function with neurodegenerative diseases (e.g., AD) without taking into consideration of the blood-brain-barrier function and the independent role of local RAS in the brain tissue.

\section{Conclusions}

Our findings first proved that lisinopril increased the promoter activity of an ACE gene in neurons, and that the I/D polymorphism of the ACE gene contrarily responds to the $\mathrm{ACE}$ inhibitors in regulating the $\mathrm{ACE}$ expression in neurons. Hitherto, our study moves an important step forward to provide a deep insight for the future pharmacogenetic testing of the ACE I/D polymorphism responding to the ACE inhibitors in AD patients.

Herein, we also propose that first it may be necessary to subgroup the same pathological type of $\mathrm{AD}$ before the researchers tackle a pharmacogenetic trial to clarify the response of an ACE I/D to ACE the inhibitors. For instance, the subgroup could be the individuals of $\mathrm{AD}$ with hypertension, $\mathrm{AD}$ with amyloid burden, or AD without macrovascular events. As doing such, it may provide new experimental evidence to guide the clinical doctors to do personalized therapy in the future.

\section{Disclosure Statement}

The authors have no actual or potential conflicts of interest to disclose. No writing assistance was utilized in the production of this manuscript.

\section{Acknowledgments}

This work was supported by research grants from Kaohsiung Medical University Hospital (KMUH102-2T105) to YH Yang, NSYSU-KMU Joint Research Project (\#NSYSUKMU 105-P022) to Hsueh-Wei Chang, and Kaohsiung Medical University (KMU-M103012) to SJ Wu. The authors would like to thank Dr. Wang Chi-Huei (Department of Biotechnology, Kaohsiung Medical University) kindly provided us the pGL4 Luciferase Reporter Vectors. The authors also thank Dr. Shui Hao-Ai (National Defense Medical Center of R.O.C., National Defense University) generously gave us SH-SY5Y cells.

\section{References}

[1] Chobanian, A. V., Bakris, G. L., Black, H. R., Cushman, W.C., Green, L. A., Izzo Jr, J. L., et al. 2003. “The Seventh Report of the Joint National Committee on Prevention, Detection, Evaluation, and Treatment of High Blood Pressure: the JNC 7 Report." JAMA: The Journal of the American Medical Association 289 (19): 2560-72.

[2] Ohrui, T., Tomita, N., Sato-Nakagawa, T., Matsui, T., Maruyama, M., Niwa, K., et al. 2004. "Effects of Brain-Penetrating ACE Inhibitors on Alzheimer Disease Progression." Neurology 63 (7): 1324-5.

[3] Rozzini, L., Chilovi, B. V., Bertoletti, E., Conti, M., Del Rio, I., Trabucchi, M., et al. 2006. "Angiotensin Converting Enzyme (ACE) Inhibitors Modulate the Rate of Progression of Amnestic Mild Cognitive Impairment." International Journal of Geriatric Psychiatry 21 (6): 550-5.

[4] Sink, K. M., Leng, X., Williamson, J., Kritchevsky, S. B., Yaffe, K., Kuller, L., et al. 2009. "Angiotensin-Converting Enzyme Inhibitors and Cognitive Decline in Older Adults with Hypertension: Results from the Cardiovascular Health Study." Archives of Internal Medicine 169 (13): 1195-202.

[5] Yasar, S., Zhou, J., Varadhan, R., and Carlson, M. C. 2008. "The Use of Angiotensin-Converting Enzyme Inhibitors and Diuretics Is Associated with a Reduced Incidence of Impairment on Cognition in Elderly Women." Clinical Pharmacology and Therapeutics 84 (1): 119-26.

[6] O'caoimh, R., Healy, L., Gao, Y., Svendrovski, A., Kerins, D. M., Eustace, J., et al. 2014. "Effects of Centrally Acting Angiotensin Converting Enzyme Inhibitors on Functional Decline in Patients with Alzheimer's Disease." Journal of Alzheimer's Disease: JAD 40 (3): 595-603.

[7] Tzourio, C., Anderson, C., Chapman, N., Woodward, M., Neal, B., MacMahon, S., et al. 2003. "Effects of Blood Pressure Lowering with Perindopril and Indapamide Therapy on Dementia and Cognitive Decline in Patients with Cerebrovascular Disease." Archives of Internal Medicine 163 (9): 1069-75.

[8] Hanon, O., and Forette, F. 2004. "Prevention of Dementia: Lessons from SYST-EUR and PROGRESS." Journal of the Neurological Sciences 226 (1-2): 71-4.

[9] Duron, E., Rigaud, A. S., Dubail, D., Mehrabian, S., Latour, F., Seux, M. L., et al. 2009. "Effects of 

Inhibitors in Regulating the ACE Promoter Activity in Neurons

Antihypertensive Therapy on Cognitive Decline in Alzheimer's Disease." American Journal of Hypertension 22 (9): 1020-4.

[10] Oveisgharan, S., and Hachinski, V. 2010. "Hypertension, Executive Dysfunction, and Progression to Dementia: The Canadian Study of Health and Aging." Archives of Neurology 67 (2): 187-92.

[11] Solfrizzi, V., Scafato, E., Frisardi, V., Seripa, D., Logroscino, G., Kehoe, P. G., et al. 2013. "Angiotensin-Converting Enzyme Inhibitors and Incidence of Mild Cognitive Impairment. The Italian Longitudinal Study on Aging." Age 35 (2): 441-53.

[12] Abdalla, S., Langer, A., Fu, X., and Quitterer, U. 2013. "ACE Inhibition with Captopril Retards the Development of Signs of Neurodegeneration in an Animal Model of Alzheimer's Disease." International Journal of Molecular Sciences 14 (8): 16917-42.

[13] Yamada, K., Horita, T., Takayama, M., Takahashi, S., Takaba, K., Nagata, Y., et al. 2011. "Effect of a Centrally Active Angiotensin Converting Enzyme Inhibitor, Perindopril, on Cognitive Performance in Chronic Cerebral Hypo-perfusion Rats.” Brain Research 1421: 110-20.

[14] Yang, Y. L., Xia, G. X., and Ye, G. 2009. "Determination of Two Metabolites of Calycosin-7-O-beta-Dglucopyranoside in Rat Urine by HPLC." Biomedical Chromatography: BMC 23 (1): 48-53.

[15] Khachaturian, A. S., Zandi, P. P., Lyketsos, C. G., Hayden, K. M., Skoog, I., Norton, M. C., et al. 2006. "Antihypertensive Medication Use and Incident Alzheimer Disease: The Cache County Study." Archives of Neurology 63 (5): 686-92.

[16] Poon, I. O. 2008. "Effects of Antihypertensive Drug Treatment on the Risk of Dementia and Cognitive Impairment." Pharmacotherapy 28 (3): 366-75.

[17] Staessen, J. A., Thijs, L., Richart, T., Odili, A. N., and Birkenhager, W. H. 2011. "Placebo-Controlled Trials of Blood Pressure-Lowering Therapies for Primary Prevention of Dementia." Hypertension 57 (2): e6-7.

[18] Teo, K., Yusuf, S., Sleight, P., Anderson, C., Mookadam, F., Ramos, B., et al. 2004. "Rationale, Design, and Baseline Characteristics of 2 Large, Simple, Randomized Trials Evaluating Telmisartan, Ramipril, and Their Combination in High-Risk Patients: The Ongoing Telmisartan Alone and in Combination with Ramipril Global Endpoint Trial/Telmisartan Randomized Assessment Study in ACE Intolerant Subjects with Cardiovascular Disease (ONTARGET/TRANSCEND) Trials." American Heart Journal 148 (1): 52-61.

[19] Harmer, D., Gilbert, M., Borman, R., and Clark, K. L. 2002. "Quantitative mRNA Expression Profiling of ACE 2, a Novel Homologue of Angiotensin Converting
Enzyme.” FEBS Letters 532 (1-2): 107-10.

[20] Sibony, M., Gasc, J. M., Soubrier, F., Alhenc-Gelas, F., and Corvol, P. 1993. "Gene Expression and Tissue Localization of the Two Isoforms of Angiotensin I Converting Enzyme." Hypertension 21 (6 Pt 1): 827-35.

[21] Arregui, A., Perry, E. K., Rossor, M., and Tomlinson, B. E. 1982. “Angiotensin Converting Enzyme in Alzheimer's Disease Increased Activity in Caudate Nucleus and Cortical Areas." Journal of Neurochemistry 38 (5): 1490-2.

[22] Miners, J. S., Ashby, E., Van Helmond, Z., Chalmers, K. A., Palmer, L. E., Love, S., et al. 2008. "Angiotensin-Converting Enzyme (ACE) Levels and Activity in Alzheimer's Disease, and Relationship of Perivascular ACE-1 to Cerebral Amyloid Angiopathy." Neuropathology and Applied neurobiology 34 (2): 181-93.

[23] Savaskan, E., Hock, C., Olivieri, G., Bruttel, S., Rosenberg, C., Hulette, C., et al. 2001. "Cortical Alterations of Angiotensin Converting Enzyme, Angiotensin II and AT1 Receptor in Alzheimer's Dementia." Neurobiology of Aging 22 (4): 541-6.

[24] Bernstein, K. E., Ong, F. S., Blackwell, W. L., Shah, K. H., Giani, J. F., Gonzalez-Villalobos, R. A., et al. 2013. "A Modern Understanding of the Traditional and Nontraditional Biological Functions of Angiotensin-Converting Enzyme." Pharmacological Reviews 65 (1): 1-46.

[25] Hu, J., Igarashi, A., Kamata, M., and Nakagawa, H. 2001. "Angiotensin-Converting Enzyme Degrades Alzheimer Amyloid Beta-peptide (A Beta); Retards A Beta Aggregation, Deposition, Fibril Formation; and Inhibits Cytotoxicity." The Journal of Biological Chemistry 276 (51): 47863-8.

[26] Oba, R., Igarashi, A., Kamata, M., Nagata, K., Takano, S., and Nakagawa, H. 2005. "The N-terminal Active Centre of Human Angiotensin-Converting Enzyme Degrades Alzheimer Amyloid Beta-peptide.” The European Journal of Neuroscience 21 (3): 733-40.

[27] Fleming, I., Kohlstedt, K., and Busse, R. 2005. "New FACEs to the Renin-Angiotensin System." Physiology 20: 91-5.

[28] Barauna, V. G., Campos, L. C., Miyakawa, A. A., and Krieger, J. E. 2011. "ACE as a Mechanosensor to Shear Stress Influences the Control of Its Own Regulation via Phosphorylation of Cytoplasmic Ser (1270)." PloS One 6 (8): e22803.

[29] Elkins, J. S., Douglas, V. C., and Johnston, S. C. 2004. "Alzheimer Disease Risk and Genetic Variation in ACE: A Meta-analysis.” Neurology 62 (3): 363-8.

[30] Kehoe, P. G., Russ, C., Mcilory, S., Williams, H., Holmans, P., Holmes, C., et al. 1999. "Variation in DCP1, Encoding ACE, Is Associated with Susceptibility to 
Alzheimer Disease." Nature Genetics 21 (1): 71-2.

[31] Lehmann, D. J., Cortina-Borja, M., Warden, D. R., Smith, A. D., Sleegers, K., Prince, J. A., et al. 2005. "Large Meta-analysis Establishes the ACE Insertion-Deletion Polymorphism as a Marker of Alzheimer's Disease." American Journal of Epidemiology 162 (4): 305-17.

[32] Mattila, K. M., Rinne, J. O., Roytta, M., Laippala, P., Pietilä, T., Kalimo, H., et al. 2000. "Dipeptidyl Carboxypeptidase 1 (DCP1) and Butyrylcholinesterase (BCHE) Gene Interactions with the Apolipoprotein E Epsilon4 Allele as Risk Factors in Alzheimer's Disease and in Parkinson's Disease with Coexisting Alzheimer Pathology." Journal of Medical Genetics 37 (10): 766-70.

[33] Meng, Y., Baldwin, C. T., Bowirrat, A., Waraska, K., Inzelberg, R., Friedland, R. P., et al. 2006. "Association of Polymorphisms in the Angiotensin-Converting Enzyme Gene with Alzheimer Disease in an Israeli Arab Community." American Journal of Human Genetics 78 (5): 871-7.

[34] Harrap, S. B., Tzourio, C., Cambien, F., Poirier, O., Raoux, S., Chalmers, J., et al. 2003. "The ACE Gene I/D Polymorphism Is Not Associated with the Blood Pressure and Cardiovascular Benefits of ACE Inhibition." Hypertension 42 (3): 297-303.

[35] Hajjar, I., Kritchevsky, S., Newman, A. B., Li, R., Yaffe, K., Simonsick, E. M., et al. 2010. "Renin Angiotensin System Gene Polymorphisms Modify Angiotensin-Converting Enzyme Inhibitors' Effect on Cognitive Function: The Health, Aging and Body Composition Study." Journal of the American Geriatrics Society 58 (6): 1035-42.

[36] Wu, S. J., Hsieh, T. J., Kuo, M. C., Tsai, M. L., Tsai, K. L., Chen, C. H., et al. 2013. "Functional Regulation of Alu Element of Human Angiotensin-Converting Enzyme Gene in Neuron Cells." Neurobiology of Aging 34 (7): e1921-7.

[37] Inoue, I., Nakajima, T., Williams, C. S., Quackenbush, J., Puryear, R., and Powers, M., et al. 1997. "A Nucleotide Substitution in the Promoter of Human Angiotensinogen Is Associated with Essential Hypertension and Affects Basal Transcription in vitro." The Journal of Clinical Investigation 99 (7): 1786-97.

[38] Costerousse, O., Allegrini, J., Clozel, J. P., Menard, J., and Alhenc-Gelas, F. 1998. "Angiotensin I-converting Enzyme Inhibition but Not Angiotensin II Suppression Alters Angiotensin I-converting Enzyme Gene Expression in Vessels and Epithelia." The Journal of Pharmacology and Experimental Therapeutics 284 (3): 1180-7.

[39] Kohlstedt, K., Brandes, R. P., Muller-Esterl, W., Busse, R., and Fleming, I. 2004. "Angiotensin-Converting enzyme Is Involved in Outside-In Signaling in Endothelial Cells." Circulation Research 94 (1): 60-7.
[40] Zineh, I., Pacanowski, M., and Woodcock, J. 2013. "Pharmacogenetics and Coumarin Dosing-Recalibrating Expectations." The New England Journal of Medicine 369 (24): 2273-5.

[41] Kehoe, P. G., and Wilcock, G. K. 2007. "Is Inhibition of the Renin-Angiotensin System a New Treatment Option for Alzheimer's Disease?" Lancet Neurology 6 (4): 373-8.

[42] Hemming, M. L., and Selkoe, D. J. 2005. "Amyloid Beta-Protein Is Degraded by Cellular Angiotensin-Converting Enzyme (ACE) and Elevated by an ACE Inhibitor." The Journal of Biological Chemistry 280 (45): 37644-50.

[43] Wright, J. W., and Harding, J. W. 2010. "The Brain RAS and Alzheimer's Disease." Experimental Neurology 223 (2): 326-33.

[44] Paul, M., Poyan Mehr, A., and Kreutz, R. 2006. "Physiology of Local Renin-Angiotensin Systems." Physiological Reviews 86 (3): 747-803.

[45] Abadir, P. M., Foster, D. B., Crow, M., Cooke, C. A., Rucker, J. J., Jain, A., et al. 2011. "Identification and Characterization of a Functional Mitochondrial Angiotensin System." Proceedings of the National Academy of Sciences of the United States of America 108 (36): 14849-54.

[46] Bernstein, K. E., Koronyo, Y., Salumbides, B. C., Sheyn, J., Pelissier, L., Lopes, D. H., et al. 2014. "Angiotensin-Converting Enzyme Overexpression in Myelomonocytes Prevents Alzheimer's-Like Cognitive Decline." The Journal of Clinical Investigation 124 (3): 1000-12.

[47] Danilov, S. M., Gordon, K., Nesterovitch, A. B., Lünsdorf, H., Chen, Z., Castellon, M., et al. 2011. "An Angiotensin I-converting Enzyme Mutation (Y465D) Causes a Dramatic Increase in Blood ACE via Accelerated ACE Shedding." PloS One 6 (10): e25952.

[48] Fagyas, M., Uri, K., Siket, I. M., Daragó, A., Boczán, J., Bányai, E., et al. 2014. "New Perspectives in the Renin-Angiotensin-Aldosterone System (RAAS) I: Endogenous Angiotensin Converting Enzyme (ACE) Inhibition." PloS One 9 (4): e87843.

[49] Fagyas, M., Uri, K., Siket, I. M., Daragó, A., Boczán, J., Bányai, E., et al. 2014. "New Perspectives in the Renin-Angiotensin-Aldosterone System (RAAS) III: Endogenous Inhibition of Angiotensin Converting Enzyme (ACE) Provides Protection against Cardiovascular Diseases." PloS One 9 (4): e93719.

[50] Chattopadhyay, S., Kessler, S. P., Colucci, J. A., Yamashita, M., Senanayake, P., and Sen, G. C. 2014. "Tissue-Specific Expression of Transgenic Secreted ACE in Vasculature Can Restore Normal Kidney Functions, but Not Blood Pressure, of Ace-/-Mice.” PloS One 9 (1): e87484. 